УДК 537.32

DOI: $10.15587 / 2313-8416.2015 .35893$

\title{
ТЕРМОЭЛЕКТРИЧЕСКИЕ КОЭФФИЦИЕНТЫ В ТРАНСПОРТНОЙ МОДЕЛИ ЛАНДАУЭРА-ДАТТЫ-ЛУНДСТРОМА
}

\author{
(C) Ю. А. Кругляк
}

С позиций концепции «снизу-вверх» транспортной модели Ландауэра -Датты-Лундстрома строго вы-ведены основные уравнения термоэлектричества с соответствующими транспортными коэффициентами для $1 D$ проводников в баллистическом режиме и для 3D проводников в диффузионном режиме с произвольной дисперсией и любого масштаба. Приведены термоэлектрические коэффициенты для $1 D$, $2 D$ и 3D проводников с параболической дисперсией в баллистическом и диффузионном режимах через стандартные интегральл Ферми-Дирака

Ключевые слова: нанофизика, наноэлектроника, молекулярная электроника, термоэлектрические коэффициенты, интеграль Ферми-Дирака

On the basis of the «bottom - up» approach of Landauer-Datta-Lundstrom transport model the basic equations of thermoelectricity with the corresponding transport coefficients for $1 D$ conductors in the ballistic regime and $3 D$ conductors in the diffusion regime with an arbitrary dispersion and for any size were strictly derived. The thermoelectric coefficients for $1 D, 2 D$, and $3 D$ semiconductors with parabolic dispersion in the ballistic and diffusive regimes are expressed through standard Fermi-Dirac integrals

Keywords: nanophysics, nanoelectronics, molecular electronics, thermoelectric coefficients, Fermi-Dirac integrals

\section{1. Введение}

Современная транспортная модель ЛандауэраДатты-Лундстрома (ЛДЛ) применительно к нано- и микроэлектронике изложена в [1, 2]. Модель ЛДЛ на многочисленных примерах обработки экспериментальных данных показала свою эффективность при вычислении проводимости и других электрофизических свойств резисторов любой размерности, любого масштаба и произвольной дисперсии, работающих в баллистическом, квазибаллистическом или диффузионном режиме как вблизи $0^{\circ} K$, так и при более высоких температурах. С позиций концепции «снизувверх» модели ЛДЛ в [3] рассмотрена физика термоэлектрических явлений Зеебека и Пельтье и качественно обсуждены закон Видемана-Франца, числа Лоренца и основные уравнения термоэлектричества с соответствующими транспортными коэффициентами.

Теперь получим строгие результаты, сформулированные без доказательства в [3], для 1D, 2D и $3 \mathrm{D}$ проводников с произвольной дисперсией и любого масштаба в режимах от баллистического до диффузионного, включая переходные режимы. С подробными выкладками покажем, как получаются термоэлектрические коэффициенты для 1D баллистического транспорта и для 3D диффузионного. Основным объектом обсуждения будут $n$-проводники, вместе с тем покажем каким образом распространить результаты на $p$-проводники. В рамках модели ЛДЛ рассмотрим биполярную проводимость, закон Видемана - Франца и числа Лоренца, соотношение Мотта.

В заключение в справочных целях приведем сводку термоэлектрических коэффициентов для 1D, $2 \mathrm{D}$ и $3 \mathrm{D}$ полупроводников с параболической дисперсией в баллистическом режиме, а также в диффузи- онном со степенным законом рассеяния, выраженных через стандартные интегралы Ферми-Дирака.

2. Обзор «Движущих сил» электрического тока Общее выражение для электрического тока $[1,2]$

$$
I=-I_{x}=\frac{2 q}{h} \int T(E) M(E)\left(f_{1}-f_{2}\right) d E
$$

утверждает, что любые причины, вызывающие различие между фермиевскими функциями контактов проводника, порождают ток, где $T(E)$ - коэффициент прохождения, а М(E) - число мод проводимости. Знак «-» в этом выражении указывает, что ток считается положительным, если он входит в контакт 2 (рис. 5 [3]) в направлении отрицательной полуоси $x$, электроны при этом движутся по проводнику слева направо от контакта 1 к контакту 2 в направлении положительной полуоси $x$.

Различают две «движущие силы», порождающих ток: разность потенциалов и разность температур контактов. Электроны переносят как заряд, так и тепло. Зарядовый ток дается уравнением (1). Чтобы получить общее выражение для термотока, рассуждаем следующим образом. Электроны внутри контакта движутся при энергии $E \approx E_{F}$. Чтобы зайти в моду проводимости $M(E)$ резистора со стороны истока $S$ электрон должен выделить $\left(E_{F}>E\right)$, а со стороны стока $D-$ поглотить $\left(E_{F}<E\right)$ тепловую энергию в количестве $\left|E-E_{F}\right|$. Выражение для термотока получается просто внесением $\left(E-E_{F}\right) / q$ под знак интеграла в (1), так что в итоге термоток

$$
I_{Q}=\frac{2}{h} \int\left(E-E_{F}\right) T(E) M(E)\left(f_{1}-f_{2}\right) d E
$$


Уравнения (1) и (2) есть общие выражения для зарядового тока и для термотока. Далее сразу перейдем к рассмотрению тока в режиме линейного отклика $f_{0}(E) \approx f_{1}(E)>f_{2}(E) \approx f_{0}(E)[1]$.

На рис. 1 показан ход фермиевских функций $f_{1}>f_{2}$ при одинаковой температуре контактов и $\Delta V=V_{2}-V_{1}>0$, так что ток положительный.

В этом случае знак тока не зависит от того, речь идет о n-проводнике или о р-проводнике. В режиме линейного отклика имеем [1]:

$$
\mathrm{f}_{1}-\mathrm{f}_{2} \approx\left(-\frac{\partial \mathrm{f}_{0}}{\partial \mathrm{E}}\right) \mathrm{q} \Delta \mathrm{V},
$$

где фермиевское окно проводимости $\left(-\partial f_{0} / \partial E\right) \approx \pm 2 k T$, охватывающее равновесное значение электрохимического потенциала $E=E_{F 0}$, «фильтрует» электроны и пропускает в проводник только те из них, энергия которых лежит в пределах окна Ферми.

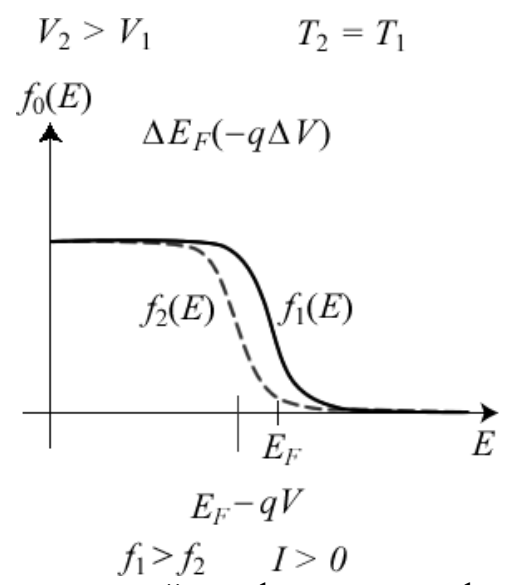

Рис. 1. Качественный ход фермиевских функций при приложении разности потенциалов к контактам, находящимся при одной и той же температуре

Рис. 2 показывает ситуацию, когда электрохимические потенциалы контактов одинаковы $\left(E_{F 1}=E_{F 2}: V_{2}=V_{1}\right)$, а температура контактов разная с $\Delta T=T_{2}-T_{1}>0$.

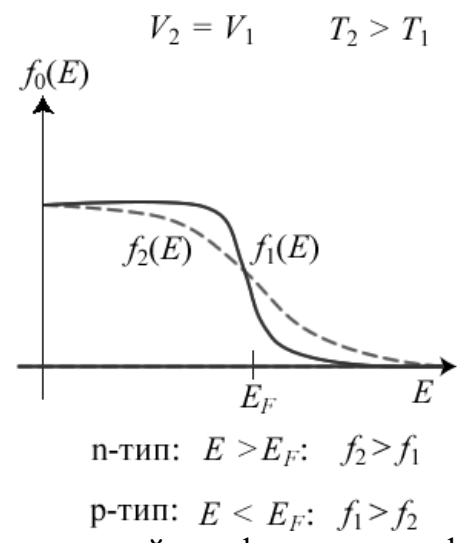

Рис. 2. Качественный ход фермиевских функций без приложения разности потенциалов к контактам,

находящимся при разных температурах

В этом случае $f_{1}>f_{2}$ для энергий электронов ниже уровня Ферми $\left(E<E_{F}\right)$ и $f_{l}<f_{2}$ для энергий элек- тронов выше уровня Ферми $\left(E>E_{F}\right)$. Направление тока зависит от того, как расположены моды проводимости проводника: если выше уровня Ферми (n-проводник), ток положительный, если же ниже уровня Ферми ( -проводник), ток отрицательный (электроны движутся по проводнику справа - налево от контакта 2 к контакту 1). В режиме линейного отклика разность фермиевских функций разложим в ряд Тейлора и ограничимся членом первого порядка:

$$
f_{1}-f_{2} \approx f_{1}-\left(f_{1}+\frac{\partial f_{1}}{\partial T} \Delta T\right)=\left(-\frac{\partial f_{0}}{\partial T}\right) \Delta T .
$$

Дифференцирование функции Ферми по температуре (ф-ла (28) в [1]) дает:

$$
f_{1}-f_{2} \approx-\left(-\frac{\partial f_{0}}{\partial E}\right) \frac{E-E_{F}}{T} \Delta T,
$$

где $T=\left(T_{1}+T_{2}\right) / 2$.

Действие обеих движущих сил - и разности электрохимических потенциалов и разности температур контактов иллюстрируется рис. 3 .

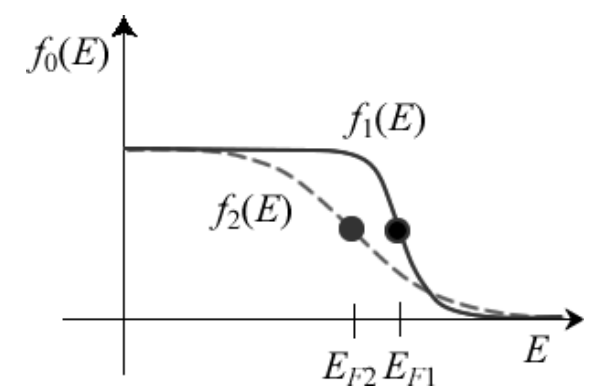

Рис. 3. Качественный ход фермиевских функций при приложении разности потенциалов к контактам,

находящимся при разных температурах

В режиме линейного отклика эффекты от обеих «сил» просто складываются:

$$
f_{1}-f_{2} \approx\left(-\frac{\partial f_{0}}{\partial E}\right) q \Delta V-\left(-\frac{\partial f_{0}}{\partial E}\right) \frac{E-E_{F}}{T} \Delta T
$$

\section{3. Электрический ток}

Окончательное выражение для суммарного тока есть сумма вкладов от всех мод проводимости:

$$
I=\int I^{\prime}(E) d E,
$$

где ток в дифференциальной форме

$$
I^{\prime}(E)=\frac{2 q}{h} T(E) M(E)\left(f_{1}-f_{2}\right) .
$$

Подставляя разность фермиевских функций через (6), получаем

$$
I^{\prime}(E)=G^{\prime}(E) \Delta V+S_{T}^{\prime}(E) \Delta T
$$

где

$$
G^{\prime}(E)=\frac{2 q^{2}}{h} T(E) M(E)\left(-\frac{\partial f_{0}}{\partial E}\right),
$$

есть дифференциальная проводимость, а 


$$
\begin{aligned}
S_{T}^{\prime}(E) & =-\frac{2 q^{2}}{h} T(E) M(E)\left(\frac{E-E_{F}}{q T}\right)\left(-\frac{\partial f_{0}}{\partial E}\right)= \\
& =-\frac{k}{q}\left(\frac{E-E_{F}}{k T}\right) G^{\prime}(E)
\end{aligned}
$$

есть коэффициент Соре термодиффузии в дифференциальной форме. Видно, что он отрицательный для энергий электронов выше энергии Ферми (n-проводники) и положительный для энергий электронов ниже энергии Ферми ( $p$-проводники).

Остается проинтегрировать дифференциальный ток (7). Окончательно получаем:

$$
I=G \Delta V+S_{T} \Delta T,
$$

где проводимость

$$
G=\int G^{\prime}(E) d E,
$$

коэффициент Соре термодиффузии

$$
\mathrm{S}_{\mathrm{T}}=\int \mathrm{S}_{\mathrm{T}}^{\prime}(\mathrm{E}) \mathrm{dE}=-\frac{\mathrm{k}}{\mathrm{q}} \int\left(\frac{\mathrm{E}-\mathrm{E}_{\mathrm{F}}}{\mathrm{kT}}\right) \mathrm{G}^{\prime}(\mathrm{E}) \mathrm{dE},[A / K] .
$$

Оба эти уравнения справедливы для проводников любой размерности и любого масштаба в режимах от баллистического через квазибаллистический до диффузионного.

\section{4. Ток в массивных проводниках}

Ток в массивных проводниках в режиме диффузионного транспорта описывается уравнениями (1)-(3) работы [3]. Из них уравнение (2) для плотности тока, выраженной через электрическое поле (3), справедливо для однородных проводников без учета коэффициента диффузии (ур-е (104) в [1]). Как переписать эти уравнения с учетом перепада температур на контактах?

Воспользуемся уравнением (12) и напомним, что в этом уравнении ток считается положительным и направлен он в сторону отрицательной полуоси $x$ (рис. 5 в [3]). Делим ток на площадь поперечного сечения проводника со знаком «минус» и получаем плотность тока в направлении положительной полуоси $x$ :

$$
J_{x}=-\frac{G}{A} \Delta V-\frac{S_{T}}{A} \Delta T .
$$

Разделим и умножим это уравнение на длину проводника $L$. Тогда

$$
J_{x}=-G \frac{L}{A} \frac{\Delta V}{L}-S_{T} \frac{L}{A} \frac{\Delta T}{L} .
$$

В диффузионном режиме

$$
G=\sigma \frac{A}{L}
$$

и по аналогии

$$
\mathrm{S}_{\mathrm{T}}=\mathrm{s}_{\mathrm{T}} \frac{\mathrm{A}}{\mathrm{L}}
$$

где $\mathbf{S}_{\mathrm{T}}$ есть удельное значение термодиффузионного коэффициента Cope. С учетом также того, что $q \Delta V / L \approx d\left(E_{F}\right) / d x$ и $\Delta T / L \approx d T / d x$, получается искомое уравнение для плотности тока в массивном проводнике с учетом градиента не только электрохимического потенциала, но и температуры:

$$
J_{x}=\sigma \frac{d\left(E_{F} / q\right)}{d x}-s_{T} \frac{d T}{d x},\left[A / M^{2}\right] .
$$

Для $n$-проводников проводимость положительна, а коэффициент Соре отрицательный. Для $p$ проводников и электронная проводимость и коэффициент Соре положительны. К подробному обсуждению проводимости в $n$ - и $p$-проводниках еще вернемся.

\section{5. Перенос тепла электронами}

Потоки тепла на контактах определяются уравнением (2). Перепищем его в дифференциальной форме

$$
I_{Q}^{\prime}(E)=\frac{2}{h}\left(E-E_{F}\right) T(E) M(E)\left(f_{1}-f_{2}\right),
$$

так что

$$
I_{Q}=\int I_{Q}^{\prime}(E) d E
$$

$\mathrm{B}$ режиме линейного отклика для разности фермиевских функций воспользуемся выражением (6), а также выражениями (10) и (11) для дифференциальных проводимости $\mathrm{G}^{\prime}(\mathrm{E})$ и коэффициента Cоре $\mathrm{S}_{\mathrm{T}}^{\prime}(\mathrm{E})$. Тогда

$$
I_{Q}^{\prime}(E)=-T S_{T}^{\prime}(E) \Delta V-K_{0}^{\prime}(E) \Delta T,
$$

где

$$
K_{0}^{\prime}(E)=\frac{\left(E-E_{F}\right)^{2}}{q^{2} T} G^{\prime}(E) .
$$
дает:

Интегрирование по всему спектру энергий

$$
I_{Q}=-T S_{T} \Delta V-K_{0} \Delta T,[B m],
$$

где

$$
K_{0}=T\left(\frac{k}{q}\right)^{2} \int\left(\frac{E-E_{F}}{k T}\right)^{2} G^{\prime}(E) d E[B m / K]
$$

есть электронная теплопроводность в условиях короткозамкнутой цепи $(\Delta V=0)$.

Итак, из общих уравнений для электрического тока (1) и термотока (2) получены соответствующие выражения (12) и (24) для режима линейного отклика. Эти выражения показывают, что различие в электрохимических потенциалах и температуре контактов ведет к электрическому току и переносу тепла электронами. Этому режиму соответствуют три транспортных параметра: проводимость $G(13)$, коэффициент термодиффузии Соре $S_{T}$ (14) и электронная теплопроводность $K_{0}(25)$. Они справедливы для проводников любой размерности и для режимов от баллистического до диффузионного. 
Для 3D-проводника в диффузионном режиме электрический ток описывается транспортным уравнением (19). Плотность теплового потока дается уравнением

$$
J_{Q x}=T s_{T} \frac{d\left(E_{F} / q\right)}{d x}-\kappa_{0} \frac{d T}{d x},\left[B m / \mathrm{M}^{2}\right]
$$

а соответствующие этим двум уравнениям (19) и (26) транспортные параметры включают удельную проводимость $\sigma$ и удельные значения термодиффузионного коэффициента Соре и удельную теплопроводность, выраженные через дифференциальную проводимость $\sigma^{\prime}(E)$ :

$$
\begin{gathered}
\sigma=\int \sigma^{\prime}(E) d E=\frac{2 q^{2}}{h}\left\langle M_{3 D}\right\rangle\langle\langle\lambda\rangle\rangle, \\
\sigma^{\prime}(E)=\frac{2 q^{2}}{h} M_{3 D}(E) \lambda(E)\left(-\frac{\partial f_{0}}{\partial E}\right),[1 / O M \cdot M \cdot Д ж] \\
\mathrm{s}_{\mathrm{T}}=-\frac{\mathrm{k}}{\mathrm{q}} \int\left(\frac{\mathrm{E}-\mathrm{E}_{\mathrm{F}}}{\mathrm{kT}}\right) \sigma^{\prime}(\mathrm{E}) \mathrm{dE},[A / \mathcal{M} \cdot K] \quad(27) \\
\kappa_{0}=T\left(\frac{k}{q}\right)^{2} \int\left(\frac{E-E_{F}}{k T}\right)^{2} \sigma^{\prime}(E) d E .[B m / \mathcal{M} \cdot K](28)
\end{gathered}
$$

Отметим, что уравнения (19) и (26) справедливы и для $1 \mathrm{D}$ и $2 \mathrm{D}$ проводников, но единицы измерения будут другими.

\section{уравнений}

\section{6. Обращенная форма транспортных}

Пары уравнений для токов (12) и (24) и для плотностей (19) и (26) получились естественным образом из общих уравнений (1) и (2) применительно к режиму линейного отклика. Они соответствуют экспериментальной ситуации, когда для заданной разности потенциалов и температур измеряются токи и потоки тепла. Если первые могут быть заданы независимо друг от друга, то потоки электронов и тепла взаимозависимы. В таком представлении вклады от всех мод проводимости складываются друг с другом. $\mathrm{C}$ точки зрения проведения экспериментов часто бывает удобным переписать эти уравнения таким образом, чтобы электрический ток и разность температур стали независимыми величинами. Обращенные таким образом уравнения (12) и (24) таковы:

$$
\begin{aligned}
& \Delta V=R I-S \Delta T, \\
& I_{Q}=-\Pi I-K \Delta T,
\end{aligned}
$$

где

$$
\begin{aligned}
& S=S_{T} / G, \\
& \Pi=T S, \\
& K=K_{0}-\Pi S G .
\end{aligned}
$$

В этом представлении вклады от разных мод проводимости не суммируются, например, сопротивление $R \neq \int R(E) d E$.

Для 3D проводника в диффузионном режиме обращение уравнений (19) и (26) дает следующую пару уравнений:

$$
\begin{gathered}
\frac{d\left(E_{F} / q\right)}{d x}=\rho J_{x}+S \frac{d T}{d x}, \\
J_{Q x}=T S J_{x}-\kappa \frac{d T}{d x}
\end{gathered}
$$

с транспортными коэффициентами

$$
\begin{gathered}
\rho=1 / \sigma, \\
S=s_{T} / \sigma, \\
\kappa=\kappa_{0}-S^{2} \sigma T .
\end{gathered}
$$

Часто можно встретить уравнение (34) с левой частью, записанной через электрическое поле.

\section{7. Транспортные коэффициенты для $1 \mathrm{D}$} проводников

Рассмотрим 1D проводник в баллистическом режиме с одной занятой подзоной. Вычислим его транспортные параметры из общих уравнений (12) и (24) или (29) и (30). В нашем случае коэффициент прохождения $T(E)=1$ и число мод проводимости $M(E)=g_{v}$, где $g_{v}$ есть долинное вырождение. Хотя мы и предполагаем, что занята только одна подзона, но в зависимости от зонной структуры $g_{v}$ может отличаться от единицы. Например, в углеродных нанотрубках, часто рассматриваемых в качестве идеальных 1D проводников, $g_{v}=2$ [4]. В случае 1D проводников все, что нам нужно знать об их зонной структуре, это только значение долинного вырождения.

Для вычисления проводимости сначала обратимся к дифференциальной проводимости (10). В нашем случае

$$
G^{\prime}(E)=\frac{2 q^{2}}{h} g_{v}\left(-\frac{\partial f_{0}}{\partial E}\right),
$$

так что проводимость

$$
G=\frac{1}{R}=\frac{2 q^{2}}{h} g_{v} \int_{E_{C}}^{\infty}\left(-\frac{\partial f_{0}}{\partial E}\right) d E=\frac{2 q^{2}}{h}\langle M\rangle .
$$

Интеграл в этом выражении есть число мод проводимости, который в случае полного вырождения $\left(T=0^{\circ} K\right)\langle M\rangle=g_{v}$. В общем же случае

$$
\langle\mathrm{M}\rangle=\mathrm{g}_{\mathrm{v}} \int_{\mathrm{E}_{\mathrm{C}}}^{\infty}\left(-\frac{\partial \mathrm{f}_{0}}{\partial \mathrm{E}}\right) \mathrm{dE}=\mathrm{g}_{\mathrm{v}} \frac{\partial}{\partial \mathrm{E}_{\mathrm{F}}} \int_{\mathrm{E}_{\mathrm{C}}}^{\infty} \mathrm{f}_{0} \mathrm{dE},
$$

где использовано свойство фермиевской функции (ф-ла (28) в [1])

$$
-\frac{\partial f_{0}}{\partial E}=+\frac{\partial f_{0}}{\partial E_{F}} .
$$

Для вычисления правого интеграла в (41)

$$
\langle M\rangle=g_{v} \frac{\partial}{\partial E_{F}} \int_{E_{C}}^{\infty} \frac{d E}{e^{\left(E-E_{F}\right) / k T}+1}
$$

сделаем замену переменных (ф-ла (16) в [2])

$$
\eta \equiv\left(E-E_{C}\right) / k T ; \quad \eta_{F} \equiv\left(E_{F}-E_{C}\right) / k T,
$$

так что 


$$
\langle M\rangle=g_{v} \frac{\partial}{\partial \eta_{F}} \int_{0}^{\infty} \frac{d \eta}{e^{\eta-\eta_{F}}+1}
$$

выражается через интеграл Ферми - Дирака [5] 0-го порядка $\mathfrak{I}_{0}\left(\eta_{F}\right)$. Дифференцирование его по параметру (ф-ла (19) в [2]) дает

$$
\langle M\rangle=g_{v} \mathfrak{I}_{-1}\left(\eta_{F}\right),
$$

так что искомая проводимость

$$
G=\frac{2 q^{2}}{h}\langle M\rangle=\frac{2 q^{2}}{h} g_{v} \mathfrak{I}_{-1}\left(\eta_{F}\right) .
$$

Для невырожденных проводников $\eta_{F} \ll 0$ и $\mathfrak{I}_{-1}\left(\eta_{F}\right) \rightarrow e^{\eta_{F}}$. Для сильно вырожденных проводников $\eta_{F} \gg 0$. Интеграл Ферми - Дирака $\mathfrak{I}_{-1}\left(\eta_{F}\right)$ как аналитическая функция

$$
\mathfrak{I}_{-1}\left(\eta_{F}\right)=\frac{\partial \mathfrak{I}_{0}\left(\eta_{F}\right)}{\partial \eta_{F}}=\frac{\partial\left[\ln \left(e^{\eta_{F}}+1\right)\right]}{\partial \eta_{F}}=\frac{e^{\eta_{F}}}{e^{\eta_{F}}+1} .
$$

Для сильно вырожденного проводника $\left(\eta_{F} \gg 0\right) \mathfrak{I}_{-1}\left(\eta_{F}\right) \rightarrow 1$ и

$$
G=\frac{2 q^{2}}{h} g_{v}
$$

как и ожидалось.

Зная $G$ и $R=1 / G$, вычислим теперь коэффициенты Соре и Зеебека. Из (14) и (39) следует, что

$$
S_{T}=-\frac{k}{q} \int_{E_{C}}^{\infty}\left(\frac{E-E_{F}}{k T}\right)\left[\frac{2 q^{2}}{h} g_{v}\left(-\frac{\partial f_{0}}{\partial E}\right)\right] d E .
$$

Разделим и умножим это выражение на проводимость согласно (40). Тогда

$$
S_{T}=-\frac{k}{q} G \frac{\int_{E_{C}}^{\infty}\left[\left(E-E_{F}\right) / k T\right]\left(-\partial f_{0} / \partial E\right) d E}{\int_{E_{C}}^{\infty}\left(-\partial f_{0} / \partial E\right) d E} .
$$

Коэффициент Соре пропорционален проводимости. Остается вычислить два интеграла. Интеграл в знаменателе равен $\mathfrak{I}_{-1}\left(\eta_{F}\right)$. Это очевидно из сравнения (46) с (41). Интеграл в числителе преобразуем следующим образом:

$$
\begin{aligned}
& \int_{E_{C}}^{\infty}\left[\left(E-E_{F}\right) / k T\right]\left(-\partial f_{0} / \partial E\right) d E= \\
= & \int_{E_{C}}^{\infty}\left[\left(E-E_{C}+E_{C}-E_{F}\right) / k T\right]\left(-\partial f_{0} / \partial E\right) d E= \\
= & \int_{E_{C}}^{\infty}\left(E-E_{C} / k T\right)\left(+\partial f_{0} / \partial E_{F}\right) d E-\eta_{F} \int_{E_{C}}^{\infty}\left(+\partial f_{0} / \partial E_{F}\right) d E,
\end{aligned}
$$

где использовалось равенство (42). Выносим $\partial / \partial E_{F}$ из под знака интеграла и делаем замену переменных (44). Тогда числитель в (51) оказывается равным

$$
\begin{aligned}
& \int_{E_{C}}^{\infty}\left[\left(E-E_{F}\right) / k T\right]\left(-\partial f_{0} / \partial E\right) d E= \\
= & \frac{\partial}{\partial \eta_{F}} \int_{0}^{\infty} \frac{\eta d \eta}{e^{\eta-\eta_{F}}+1}-\eta_{F} \frac{\partial}{\partial \eta_{F}} \int_{0}^{\infty} \frac{d \eta}{e^{\eta-\eta_{F}}+1}=. \\
= & \mathfrak{J}_{0}\left(\eta_{F}\right)-\eta_{F} \mathfrak{J}_{-1}\left(\eta_{F}\right)
\end{aligned}
$$

Окончательно коэффициент Соре (51)

$$
S_{T}=-\frac{k}{q}\left(-\eta_{F}+\frac{\mathfrak{I}_{0}\left(\eta_{F}\right)}{\mathfrak{I}_{-1}\left(\eta_{F}\right)}\right) G .
$$

Поскольку коэффициенты Соре и Зеебека связаны друг с другом через проводимость соотношением (31), то коэффициент Зеебека

$$
S=-\frac{k}{q}\left(-\eta_{F}+\frac{\mathfrak{I}_{0}\left(\eta_{F}\right)}{\mathfrak{I}_{-1}\left(\eta_{F}\right)}\right)
$$

или иначе

$$
S=-\frac{k}{q}\left(\frac{E_{C}-E_{F}}{k T}+\delta\right),
$$

где

$$
\delta=\frac{\mathfrak{I}_{0}\left(\eta_{F}\right)}{\mathfrak{J}_{-1}\left(\eta_{F}\right)} .
$$

Коэффициент Зеебека получился таким же, как и ожидалось согласно (15) в [3] с

$$
\delta=\Delta / k T .
$$

В невырожденном пределе $\left(\eta_{F} \ll 0\right)$ интегралы Ферми-Дирака становятся просто экспонентами с $\delta \rightarrow 1$ и $\Delta \rightarrow k T$.

B невырожденном 1D баллистическом резисторе ток идет по единственной моде с энергией приблизительно на $\mathrm{kT}$ выше дна зоны проводимости. Для сильно вырожденных резисторов $\left(\eta_{F} \gg 0\right)$ $\delta \rightarrow \eta_{F}=\left(E_{F}-E_{C}\right) / k T$, так что согласно (56) $S \rightarrow 0$, а $\Delta \rightarrow\left(E_{F}-E_{C}\right)$, что намного больше кванта тепловой энергии $k T$, характерного для невырожденных резисторов.

Итак, для 1D баллистического резистора вычислены $G, R, S_{T}, S, \Pi$. Перейдем к вычислению электронной теплопроводности $K_{0}$ и $K$. Следуем той же процедуре, что и при вычислении коэффициента Соре. После умножения и деления теплопроводности $K_{0}(25)$ на проводимость (40) имеем:

$$
K_{0}=T\left(\frac{k}{q}\right)^{2} G \frac{\int_{E_{C}}^{\infty}\left[\left(E-E_{F}\right) / k T\right]^{2}\left(-\partial f_{0} / \partial E\right) d E}{\int_{E_{C}}^{\infty}\left(-\partial f_{0} / \partial E\right) d E} .
$$

Сразу видим, что теплопроводность пропорциональна проводимости (закон Видемана - Франца). Как и в коэффициенте Cope (51), интеграл в знамена- 
теле равен $\mathfrak{I}_{-1}\left(\eta_{F}\right)$. Остается разобраться с интегралом в числителе. Он равен

$$
\begin{aligned}
& \int_{E_{C}}^{\infty}\left[\left(E-E_{F}\right) / k T\right]^{2}\left(-\partial f_{0} / \partial E\right) d E= \\
& =\int_{E_{C}}^{\infty}\left[\left(E-E_{C}\right)+\left(E_{C}-E_{F}\right) / k T\right]^{2}\left(-\partial f_{0} / \partial E\right) d E= \\
& =\int_{E_{C}}^{\infty}\left[\left(E-E_{C}\right) / k T\right]^{2}\left(+\partial f_{0} / \partial E_{F}\right) d E- \\
& -2 \eta_{F} \int_{E_{C}}^{\infty}\left[\left(E-E_{C}\right) / k T\right]\left(+\partial f_{0} / \partial E_{F}\right) d E+ \\
& +\left(\eta_{F}\right)^{2} \int_{E_{C}}^{\infty}\left(+\partial f_{0} / \partial E_{F}\right) d E .
\end{aligned}
$$

Последнее слагаемое есть $\left(\eta_{F}\right)^{2} \Im_{-1}\left(\eta_{F}\right)$, втоpoе слагаемое равно $-2 \eta_{F} \mathfrak{J}_{0}\left(\eta_{F}\right)$, а первое есть $2 \Im_{1}\left(\eta_{F}\right)$, так что электронная теплопроводность в условиях короткозамкнутой цепи

$$
K_{0}=T\left(\frac{k}{q}\right)^{2} G\left[2 \frac{\mathfrak{J}_{1}\left(\eta_{F}\right)}{\mathfrak{J}_{-1}\left(\eta_{F}\right)}-2 \eta_{F} \frac{\mathfrak{J}_{0}\left(\eta_{F}\right)}{\mathfrak{J}_{-1}\left(\eta_{F}\right)}+\left(\eta_{F}\right)^{2}\right] .
$$

Электронная теплопроводность в условиях незамкнутой цепи $K$ дается выражением (33) и с учетом (32) и (55) окончательно имеем:

$$
K=T\left(\frac{k}{q}\right)^{2} G\left[2 \frac{\mathfrak{I}_{1}\left(\eta_{F}\right)}{\mathfrak{I}_{-1}\left(\eta_{F}\right)}-\left(\frac{\mathfrak{I}_{0}\left(\eta_{F}\right)}{\mathfrak{I}_{-1}\left(\eta_{F}\right)}\right)^{2}\right] .
$$

Аналогичным образом можно получить аналитические выражения для термоэлектрических параметров для резисторов любой размерности, любого масштаба и любых режимов работы. Если подобные выкладки кажутся слишком скучными, вполне можно прибегнуть к численному интегрированию, что обычно достаточно для сравнения с экспериментальными данными.

В заключение настоящего обзора приведем окончательные аналитические выражения для термоэлектрических коэффициентов резисторов с параболической дисперсией всех размерностей, в том числе и для случая диффузионного режима со степенным законом рассеяния

$$
\lambda(\mathrm{E})=\lambda_{0}\left(\frac{\mathrm{E}-\mathrm{E}_{\mathrm{C}}}{\mathrm{kT}}\right)^{\mathrm{r}} .
$$

Аналогичные аналитические выражения приведем и для линейной дисперсии в графене, которому будет посвящена отдельная публикация.

\section{8. Закон Видемана-Франца и числа Лоренца}

Электроны переносят как заряд, так и тепло. Выпишем связь между электронной проводимостью и теплопроводностью в явном виде. Для этого нам потребуется процедура усреднения по удельной проводимости

$$
\sigma=\int \sigma^{\prime}(\mathrm{E}) \mathrm{dE},
$$

$$
\langle(*)\rangle \equiv \frac{\int(*) \sigma^{\prime}(\mathrm{E}) \mathrm{dE}}{\int \sigma^{\prime}(\mathrm{E}) \mathrm{dE}} .
$$

Умножаем и делим удельное значение коэффициента Cope (27) на удельную проводимость. Тогда

$$
s_{T}=-\left(\frac{k}{q}\right) \sigma\left\langle\frac{E-E_{F}}{k T}\right\rangle .
$$

Аналогично поступаем с коэффициентом Зеебека (37). Тогда с учетом (14) из работы [3]

$$
S=\frac{S_{T}}{\sigma}=-\left(\frac{k}{q}\right)\left\langle\frac{E-E_{F}}{k T}\right\rangle=-\left(\frac{k}{q}\right) \frac{E_{a v}-E_{F}}{k T}
$$

и мы видим, что коэффициент Зеебека пропорционален среднему значению энергии выше уровня Ферми, при которой течет ток.

Для удельной теплопроводности (28) и (38) имеем:

$$
\begin{gathered}
\kappa_{0}=T\left(\frac{k}{q}\right)^{2} \sigma\left\langle\left(\frac{E-E_{F}}{k T}\right)^{2}\right\rangle, \\
\kappa=T\left(\frac{k}{q}\right)^{2} \sigma\left[\left\langle\left(\frac{E-E_{F}}{k T}\right)^{2}\right\rangle-\left\langle\frac{E-E_{F}}{k T}\right\rangle^{2}\right] .
\end{gathered}
$$

Обе теплопроводности пропорциональны электронной проводимости. Отсюда

$$
\frac{\kappa}{T \sigma} \equiv L=\left(\frac{k}{q}\right)^{2}\left[\left\langle\left(\frac{E-E_{F}}{k T}\right)^{2}\right\rangle-\left\langle\frac{E-E_{F}}{k T}\right\rangle^{2}\right]
$$

есть закон Видемана-Франца, а $L$ - число Лоренца. Множитель в квадратных скобках зависит от профиля зон, вырожденности и характера рассеяния. Для типичного полупроводника с параболической дисперсией и с постоянным средним значением длины свободного пробега этот множитель приблизительно равен 2 для невырожденного проводника или $\pi^{2} / 3$ для вырожденного [3].

\section{9. Полупроводники р-типа и биполярная} проводимость

Рассмотрим 3D полупроводник с параболической дисперсией. Для зоны проводимости

$$
M_{3 D}(E)=g_{v} \frac{m^{*}}{2 \pi \hbar^{2}}\left(E-E_{C}\right), \quad\left(E \geq E_{C}\right)
$$

а для валентной зоны

$$
M_{3 D}^{(v)}(E)=g_{v} \frac{m_{p}^{*}}{2 \pi \hbar^{2}}\left(E_{V}-E\right) . \quad\left(E \leq E_{V}\right) .
$$

Проводимость обеспечивается двумя вкладами. Со стороны зоны проводимости

$$
\sigma=\frac{q^{2}}{h} \int_{E_{C}}^{\infty} M_{3 D}(E) \lambda(E)\left(-\frac{\partial f_{0}}{\partial E}\right) d E
$$

и со стороны валентной зоны

а именно: 


$$
\sigma_{p}=\frac{q^{2}}{h} \int_{-\infty}^{E_{V}} M_{3 D}^{v}(E) \lambda_{p}(E)\left(-\frac{\partial f_{0}}{\partial E}\right) d E .
$$

Коэффициент Зеебека для электронов зоны проводимости следует из (27):

$$
\begin{aligned}
& \sigma=\int_{E_{C}}^{\infty} \sigma^{\prime}(E) d E, \\
& \sigma^{\prime}(E)=\frac{2 q^{2}}{h} M_{3 D}\left(E-E_{C}\right) \lambda(E)\left(-\frac{\partial f_{0}}{\partial E}\right), \\
& S_{T}=-\frac{k}{q} \int_{E_{C}}^{\infty}\left(\frac{E-E_{F}}{k T}\right) \sigma^{\prime}(E) d E, \\
& S=S_{T} / \sigma
\end{aligned}
$$

и по аналогии для электронов валентной зоны

$$
\begin{aligned}
& \sigma_{p}=\int_{-\infty}^{E_{V}} \sigma_{p}^{\prime}(E) d E, \\
& \sigma_{p}^{\prime}(E)=\frac{2 q^{2}}{h} M_{3 D}^{v}\left(E_{V}-E\right) \lambda_{p}(E)\left(-\frac{\partial f_{0}}{\partial E}\right), \\
& S_{T}^{(v)}=-\frac{k}{q} \int_{-\infty}^{E_{V}}\left(\frac{E-E_{F}^{(p)}}{k T}\right) \sigma_{p}^{\prime}(E) d E, \\
& S_{p}=S_{T}^{(v)} / \sigma_{p},
\end{aligned}
$$

причем знак $S_{p}$ положительный.

В чем проявляются особенности когда обе зоны дают вклад в проводимость? Это встречается в узкозонных полупроводниках или при высоких температурах. В этом случае мы просто интегрируем по всем модам проводимости:

$$
\begin{array}{r}
\sigma^{t o t} \equiv \sigma+\sigma_{p}=\frac{q^{2}}{h} \int_{E_{1}}^{E_{2}} M_{3 D}^{t o t}(E) \lambda(E)\left(-\frac{\partial f_{0}}{\partial E}\right) d E, \\
M_{3 D}^{t o t}(E)=M_{3 D}(E)+M_{3 D}^{v}(E) ;
\end{array}
$$

при этом нет нужды заботиться об интегрировании до верхнего предела зоны проводимости или от самого дна валентной зоны, поскольку фермиевские функции гарантируют экспоненциальное падение до нуля на границах зон. Биполярная проводимость дается суммой вкладов от обеих мод проводимости с соответствующими значениями $\lambda(\mathrm{E})$ в нужных нам пределах энергий (рис. 4).

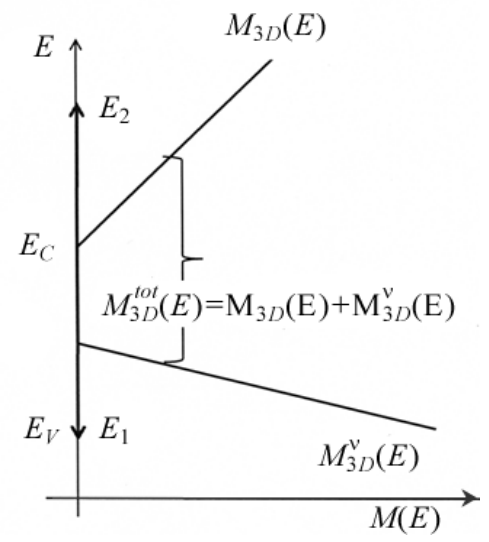

Рис. 4. К вычислению биполярной проводимости
Биполярная проводимость $\sigma^{\text {tot }}$ физически обеспечивается электронами и описывается общим уравнением (77): нет нужды менять знаки для валентной зоны или заменять $f_{0}$ на $1-f_{0}$.

Каким будет коэффициент Зеебека в режиме биполярной проводимости?

Вспомним, что при вычислении транспортных коэффициентов вклады от всех мод проводимости складываются. Для коэффициента Соре в случае биполярной проводимости имеем:

$$
s_{T}^{\text {tot }}=-\frac{k}{q} \int_{-\infty}^{+\infty}\left(\frac{E-E_{F}}{k T}\right) \sigma^{\prime}(E) d E=S \sigma+S_{p} \sigma_{p},
$$

тогда коэффициент Зеебека в режиме биполярной проводимости

$$
S^{t o t}=\frac{S \sigma+S_{p} \sigma_{p}}{\sigma+\sigma_{p}}
$$

коэффициенты Зеебека для электронов валентной зоны и зоны проводимости имеют противоположные знаки, так что в режиме биполярной проводимости суммарный коэффициент Зеебека близок к нулю и добротность [3] ТЭ устройства резко падает.

\section{0. Транспортные коэффициенты для 3D} проводников в диффузионном режиме

В заключение получим транспортные коэффициенты (36) - (38) для массивных 3D диффузионных резисторов для транспортных уравнений (34) и (35). Начнем с удельного сопротивления (36). Удельную проводимость

$$
\sigma=\frac{2 q^{2}}{h} \int_{E_{C}}^{\infty} M_{3 D}(E) \lambda(E)\left(-\frac{\partial f_{0}}{\partial E}\right) d E
$$

перепишем следующим образом:

$$
\sigma=\frac{2 q^{2}}{h}\left[\frac{\int_{E_{C}}^{\infty} M_{3 D}(E) \lambda(E)\left(-\frac{\partial f_{0}}{\partial E}\right) d E}{\int_{E_{C}}^{\infty} M_{3 D}(E)\left(-\frac{\partial f_{0}}{\partial E}\right) d E}\right] \int_{E_{C}}^{\infty} M_{3 D}(E)\left(-\frac{\partial f_{0}}{\partial E}\right) d E .
$$

Знаменатель есть среднее число мод в фермиевском окне проводимости вблизи уровня Ферми

$$
\int_{E_{C}}^{\infty} M_{3 D}(E)\left(-\frac{\partial f_{0}}{\partial E}\right) d E \equiv\left\langle M_{3 D}\right\rangle .
$$

Выражение в квадратных скобках

$$
\frac{\int_{E_{C}}^{\infty} M_{3 D}(E) \lambda(E)\left(-\frac{\partial f_{0}}{\partial E}\right) d E}{\int_{E_{C}}^{\infty} M_{3 D}(E)\left(-\frac{\partial f_{0}}{\partial E}\right) d E}=\frac{\left\langle M_{3 D} \lambda(E)\right\rangle}{\left\langle M_{3 D}\right\rangle} \equiv\langle\langle\lambda\rangle\rangle
$$

есть средняя длина свободного пробега, усредненная по всем модам в окне проводимости. Таким образом, вычисление удельной проводимости 3D диффузионного резистора сводится к вычислению среднего числа мод в окне проводимости и усредненного по этим модам средней длины свободного пробега: 


$$
\sigma=\frac{2 q^{2}}{h}\left\langle M_{3 D}\right\rangle\langle\langle\lambda\rangle\rangle .
$$

Для параболической дисперсии (71) из (82) имеем:

$$
\begin{aligned}
& \left\langle M_{3 D}\right\rangle=g_{v} \frac{m^{*}}{2 \pi \hbar^{2}} k T \int_{E_{C}}^{\infty}\left(\frac{E-E_{C}}{k T}\right)\left(-\frac{\partial f_{0}}{\partial E}\right) d E= \\
& =M_{3 D}(k T) \int_{E_{C}}^{\infty} \eta\left(+\frac{\partial f_{0}}{\partial \eta_{F}}\right) d \eta=M_{3 D}(k T) \mathfrak{I}_{0}\left(\eta_{F}\right)
\end{aligned}
$$

где $M_{3 D}(k T)$ это выражение (71), вычисленное при $E-E_{C}=k T$, а новые переменные определены в (44).

Усредненное значение $\langle\lambda\rangle$ по (83) вычислим для степенного закона рассеяния (63), в котором показатель степени $r$ принимает разные значения для разных механизмов рассеяния [6]. Числитель в (83) преобразуется следующим образом:

$$
\begin{aligned}
& \int_{\mathrm{E}_{\mathrm{C}}}^{\infty} \mathrm{M}_{3 \mathrm{D}}(\mathrm{E}) \lambda(\mathrm{E})\left(-\frac{\partial \mathrm{f}_{0}}{\partial \mathrm{E}}\right) \mathrm{dE}= \\
& =\int_{\mathrm{E}_{\mathrm{C}}}^{\infty} \mathrm{g}_{\mathrm{v}} \frac{\mathrm{m}^{*}}{2 \pi \hbar^{2}}\left(\mathrm{E}-\mathrm{E}_{\mathrm{C}}\right) \lambda_{0}\left(\frac{\mathrm{E}-\mathrm{E}_{\mathrm{C}}}{\mathrm{kT}}\right)^{\mathrm{r}}\left(-\frac{\partial \mathrm{f}_{0}}{\partial \mathrm{E}}\right) \mathrm{dE}=. \\
& =\mathrm{M}_{3 \mathrm{D}}(\mathrm{kT}) \lambda_{0} \Gamma(\mathrm{r}+2) \mathfrak{J}_{\mathrm{r}}\left(\eta_{\mathrm{F}}\right)
\end{aligned}
$$

Поскольку знаменатель в (83) есть $\left\langle\mathrm{M}_{3 \mathrm{D}}\right\rangle$ по (85), то

$$
\langle\langle\lambda\rangle\rangle=\lambda_{0} \Gamma(r+2) \frac{\mathfrak{I}_{r}\left(\eta_{F}\right)}{\mathfrak{J}_{0}\left(\eta_{F}\right)},
$$

а проводимость (84) с учетом (85)

$$
\sigma=\frac{2 q^{2}}{h} M_{3 D}(k T) \mathfrak{I}_{0}\left(\eta_{F}\right)\left[\lambda_{0} \Gamma(r+2) \frac{\mathfrak{J}_{r}\left(\eta_{F}\right)}{\mathfrak{J}_{0}\left(\eta_{F}\right)}\right] .
$$

Итак, вычислили удельную проводимость и далее удельное сопротивление $\rho$ по (36). Теперь вычислим коэффициент Зеебека по (37) и (27). С учетом (63) коэффициент Зеебека дается уравнением (56) с

$$
\delta=\frac{\int\left(\frac{E-E_{C}}{k T}\right) \sigma^{\prime}(E) d E}{\int \sigma^{\prime}(E) d E}=(r+2) \frac{\mathfrak{I}_{r+1}\left(\eta_{F}\right)}{\mathfrak{I}_{r}\left(\eta_{F}\right)} .
$$

Более общее определение этого параметра есть (формула (25) в [3])

$$
\delta=\left(\mathrm{E}_{\mathrm{av}}-\mathrm{E}_{\mathrm{C}}\right) / \mathrm{kT} .
$$

Рассмотрим невырожденный предел $\left(\eta_{\mathrm{F}} \ll 0\right)$. Оба интеграла Ферми - Дирака в (89) становятся просто $e^{\eta_{F}}$ и $\delta=r+2$. При $r=0$ параметр $\delta=2$ и согласно (90) это означает, что ток течет при средней энергии на $2 k T$ выше дна зоны проводимости $E_{C}$. При $r>0$ средняя длина свободного пробега (63) растет с ростом энергии, в результате чего ток течет при более высокой энергии и соответственно увеличивается коэффициент Зеебека. Для $r=2$, что характерно для рассеяния на ионизированных примесях, $\delta=4$ и ток течет при средней энергии на $4 k T$ выше $\mathrm{E}_{\mathrm{C}}$.

Остается вычислить удельную теплопроводность (38) или иначе число Лоренца (70):

$$
\mathrm{L}=\frac{\mathrm{K}}{\mathrm{T} \sigma}=\left(\frac{\mathrm{k}}{\mathrm{q}}\right)^{2}\left\{(\mathrm{r}+2)(\mathrm{r}+3) \frac{\mathfrak{I}_{\mathrm{r}+2}\left(\eta_{\mathrm{F}}\right)}{\mathfrak{I}_{\mathrm{r}}\left(\eta_{\mathrm{F}}\right)}-\left[(\mathrm{r}+2) \frac{\mathfrak{I}_{\mathrm{r}+1}\left(\eta_{\mathrm{F}}\right)}{\mathfrak{J}_{\mathrm{r}}\left(\eta_{\mathrm{F}}\right)}\right]^{2}\right\}
$$

Этот частный результат полезно сравнить с общим выражением (70), которое не предполагает параболическую дисперсию и степенной закон рассеяния. В невырожденном пределе $r=0$ и выражение в фигурных скобках есть просто 2 как и ожидалось (ф-ла (22) в [3]). В вырожденном пределе $\left(\eta_{\mathrm{F}} \gg 0\right)$ можно воспользоваться разложением интегралов Ферми-Дирака в ряд [5] и показать, что выражение в фигурных скобках равно $\pi^{2} / 3$.

\section{1. Коэффициент Зеебека в вырожденном пределе: формула Мотта}

Коэффициент Зеебека (56) с параметром $\delta$ по (89) справедлив для параболической дисперсии и степенного закона рассеяния. В невырожденном пределе интегралы Ферми-Дирака становятся просто экспонентой, в результате чего выражение для коэффициента Зеебека упрощается до

$$
S=-\left(\frac{k}{q}\right)\left[(r+2)-\eta_{F}\right] .
$$

Упростить коэффициент Зеебека можно и в сильно вырожденном пределе $\left(\eta_{F} \gg 0\right)$ следующим образом. В этом пределе интеграл Ферми - Дирака порядка $r$ аппроксимируется рядом [5]

$$
\mathfrak{I}_{r}\left(\eta_{F}\right) \approx \frac{\left(\eta_{F}\right)^{r+1}}{\Gamma(r+2)}+\frac{\left(\eta_{F}\right)^{r-1}}{\Gamma(r)} \varsigma(2)+\ldots,
$$

где $\varsigma(2)=\pi^{2} / 6$ есть дзета-функция Римана. Воспользуемся этим разложением для коэффициента Зеебека (56) с параметром $\delta$ по (89)

$$
S=-\frac{k}{q}\left[(r+2) \frac{\mathfrak{I}_{r+1}\left(\eta_{F}\right)}{\mathfrak{J}_{r}\left(\eta_{F}\right)}-\eta_{F}\right]
$$

и найдем

$$
S \approx-\frac{k}{q}\left[\frac{2(r+1) \eta_{F} \varsigma(2)}{\left(\eta_{F}\right)^{2}+r(r+1) \varsigma(2)}\right],
$$

что в сильно выраженном пределе $\left(\eta_{F} \gg 0\right)$ дает

$$
S \approx-\frac{k}{q}\left[\frac{2(r+1) \varsigma(2)}{\eta_{F}}\right] .
$$

Мы видим из ур-я (92), что в невырожденном пределе

$$
|S| \propto-\eta_{F},\left(\eta_{F} \ll 0\right),
$$

а из уравнения (96) в вырожденном пределе

$$
|S| \propto 1 / \eta_{F} .\left(\eta_{F} \ll 0\right) .
$$

Наконец, вспомним, что

$$
\sigma^{\prime}(E) \propto M_{3 D}(E) \lambda(E) \propto\left(E-E_{C}\right)^{r+1},
$$

откуда 


$$
\left.\frac{1}{\sigma^{\prime}(\mathrm{E})} \frac{\mathrm{d} \sigma^{\prime}(\mathrm{E})}{\mathrm{dE}}\right|_{\mathrm{E}=\mathrm{E}_{\mathrm{F}}}=\frac{\mathrm{r}+1}{\mathrm{E}_{\mathrm{F}}-\mathrm{E}_{\mathrm{C}}} .
$$

Подставляя в (96) $\varsigma(2)$ и $r+1$ из (100) получаем, наконец, известное соотношение Мотта для коэффициента Зеебека

$$
S \approx-\frac{\pi^{2} k^{2} T}{3 q}\left[\frac{\partial \ln \sigma^{\prime}(E)}{\partial E}\right]_{E=E_{F}},
$$

которое широко используется для вырожденных полупроводников. Для получения этого соотношения мы воспользовались уравнением (94), для которого предполагается параболическая зонная структура и степенной закон рассеяния. Этот же результат можно получить и из более общих соображений, если воспользоваться известным разложением Зоммерфельда $[7,8]$.

\section{2. Анализ экспериментальных данных для} Ge: коэффициент Зеебека и термоэлектрические коэффициенты

На рис. 5 показана зависимость коэффициентов Зеебека для Ge n- и p-типа при комнатной температуре в зависимости от положения уровня Ферми $E_{F}$ относительно дна зоны проводимости $E_{C}$.

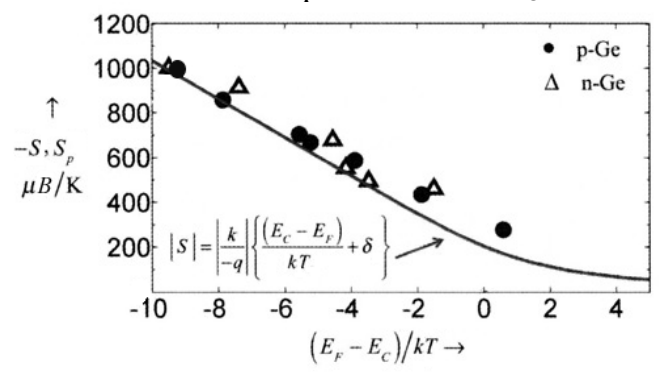

Рис. 5. Коэффициент Зеебека для $\mathrm{Ge} \mathrm{n}$ - и р-типа при $T=300 \mathrm{~K}$ : экспериментальные точки по [9], расчетная кривая согласно [6]

Покажем, что экспериментальные результаты согласуются с уравнением (56) и указывают на то, что поправка $\delta(58)$ мала и постоянна у невырожденных проводников. Покажем также, что поправка $\delta$ растет по мере удаления уровня Ферми от дна зоны проводимости. Рассмотрим также ситуацию с р-проводниками.

Согласно (56) коэффициент Зеебека $|S|$ должен падать линейно с ростом $E_{F}$, если только поправка $\delta(58)$ постоянна. Этот вывод подтверждается экспериментальными данными (рис. 5). Так

$$
|S|=86 \times\left(-\eta_{F}+\delta\right),[\mu B / K]
$$

где $\eta_{F}$ определяется по (44). При $\eta_{F}=-10$ величина $|S| \approx 1000 \mu B / K$, откуда следует, что $\delta \approx 2$ в соответствии с приведенными выше простыми рассуждениями для невырожденных проводников.

Если бы поправка $\delta$ была всегда постоянна, то коэффициент Зеебека менял бы знак при $E_{F}>E_{c}+2 k T$. На самом деле этого не происходит (рис. 5), что указывает на то, что поправка $\delta$ должна увеличиваться по мере того как уровень Ферми поднимается все выше по зоне проводимости. При $\eta_{F}=4$ коэффициент Зеебека $|S| \approx 50 \mu \mathrm{B} /$ К (рис. 5), что соответствует $\delta \approx 4.6$, а это в два раза превышает значение этой поправки для невырожденных условий. Как было показано в [3], поправка

$$
\delta=\left(E_{a v}-E_{c}\right) / k T,
$$

где $E_{a v}$ есть среднее значение энергии, при которой движутся носители тока, показывает нам на каком удалении от дна зоны проводимости движутся электроны. У сильно вырожденных проводников $E_{a v} \rightarrow E_{F}$, так что $\delta \rightarrow \eta_{F}$ и $S \rightarrow 0$ при $E_{F} \gg E_{c}$.

Для n-проводников коэффициент Зеебека (по модулю) тем больше, чем ниже уровень Ферми находится по отношению к дну зоны проводимости. Знак коэффициента Зеебека отрицательный. В случае рпроводников коэффициент Зеебека тем больше, чем выше уровень Ферми находится по отношению к потолку валентной зоны и знак его положительный. Токовые уравнения (4) и (5) для n-проводников в [3] при переходе к р-проводникам не изменяются, а коэффициент Зеебека запишется следующим образом:

$$
\begin{aligned}
& \frac{d\left(E_{F}^{(p)} / q\right)}{d x}=\rho_{p} J_{p x}+S_{p} \frac{d T}{d x}, \\
& S_{p}(T)=\left(\frac{k}{+q}\right)\left(\frac{E_{F}^{(p)}-E_{v}}{k T}+\delta_{p}\right),
\end{aligned}
$$

где $\delta_{p}=\left(E_{v}-E_{a v}\right) / k T$ является положительным числом, которое показывает нам насколько ниже потолка валентной зоны движутся «дырки». Как мы уже видели выше, в условиях биполярной проводимости и «дырки» и электроны дают вклад в коэффициент Зеебека. Учет этого обстоятельства становится важным при высоких температурах и для узкощелевых полупроводников.

Обратимся к термоэлектрическим коэффициентам для Ge. Оценим их для слаболегированного $\mathrm{n}$ $\mathrm{Ge}$ при комнатной температуре. Для него известно, что при $T=300 K$ подвижность $\mu=3200 \mathrm{~cm}^{2} / B \cdot c$, а равновесная плотность носителей тока $n_{0} \approx N_{D}=10^{-15} \mathrm{~cm}^{-3}$ [10]. Вспомним также, что для невырожденных полупроводников справедливо простое соотношение между плотностью носителей тока и положением уровня Ферми, а именно [10]:

$$
n_{0}=N_{c} e^{\left(E_{F}-E_{c}\right) / k T},
$$

где «эффективная плотность состояний» для $\mathrm{Ge}$ при комнатной температуре $N_{c}=1.09 \times 10^{19} \mathrm{cM}^{-3}[10]$.

Основные уравнения термоэлектричества для массивного образца даются (34) и (35) с четырьмя транспортными коэффициентами: удельное сопротивление $\rho$ (36), коэффициент Зеебека $S$ (37), электронная теплопроводность $\kappa$ (38) и коэффициент Пельтье $\pi=T S$ (соотношение Кельвина).

Имеющиеся в нашем распоряжении данные позволяют сразу оценить удельную проводимость $\sigma=n_{0} q \mu$, откуда удельное сопротивление $\rho \approx 2 \Omega \cdot c M$.

Коэффициент Зеебека дается соотношением (56) и он зависит от положения уровня Ферми. Из (105) имеем 


$$
\frac{E_{c}-E_{F}}{k T}=\ln \frac{N_{c}}{n_{0}} \approx 9.3 .
$$

В предположении, что $\delta=2$, для коэффициента Зеебека получаем $S \approx-970$ мк $B / K$.

Коэффициент Пельтье находим из соотношения Кельвина: $\pi=-0.3 \mathrm{Bm} / \mathrm{A}$.

Электронную теплопроводность найдем через число Лоренца [3]:

$$
\kappa=T \sigma L .
$$

Поскольку в нашем случае речь идет о слаболегированном 3D полупроводнике с почти параболической зонной структурой, то мы вправе предположить [3], что число Лоренца как для невырожденного проводника $L \approx 2(k / q)^{2}$, откуда $\kappa \approx 2.2 \times 10^{-4} \mathrm{Bm} / \mathrm{M} \cdot K$.

Рассмотрен довольно простой пример. Полезно продумать что изменится в этой сводке термоэлектрических коэффициентов $\mathrm{Ge}$, если температура понизится до $77 K$ или температура останется комнатной, а легирование возрастет до $10^{20} \mathrm{~cm}^{3}$. Нужно помнить, что решетка также проводит тепло. Решеточная теплопроводность Ge при комнатной температуре достигает $58 \mathrm{Bm} / \mathrm{M} \cdot K$, что на пять порядков больше оцененной выше электронной теплопроводности. В сильнолегированных полупроводниках с низкой решеточной теплопроводностью вклад электронов в теплопроводность может быть существенным.

Подведем итоги. В нашу задачу входило получить строгие результаты, сформулированные без доказательства в [3], для 1D, 2D и 3D проводников с произвольной дисперсией и любого масштаба в режимах от баллистического до диффузионного. Показано с подробными выкладками как получаются термоэлектрические коэффициенты для 1D баллистического транспорта и для 3D диффузионного. Основным объектом обсуждения были $n$-проводники, вместе с тем мы показали каким образом распространить результаты на $p$-проводники. Рассмотрели биполярную проводимость, закон Видемана - Франца и числа Лоренца, соотношение Мотта. Процессы рассеяния учитывали феноменологически. В коэффициенте прохождения $T(E)=\lambda(E) /[\lambda(E)+L]$ среднюю длину свободного пробега описывали степенным законом.

В заключение в справочных целях собраны воедино в Приложении термоэлектрические коэффициенты для 1D, 2D и 3D полупроводников с параболической дисперсией $[6,11]$.

Приложение. Термоэлектрические коэффициенты для 1D, 2D и 3D проводников с параболической дисперсией

В приведенных ниже формулах предполагается параболическая зонная структура

$$
E(k)=\frac{\hbar^{2} k^{2}}{2 m^{*}}
$$

и степенной закон рассеяния

$$
\lambda(E)=\lambda_{0}\left(\frac{E}{k T}\right)^{r} .
$$

Положение уровня Ферми $E_{F}$ относительно дна зоны проводимости $E_{C}$ определяется параметром

$$
\eta_{F}=\frac{E_{F}-E_{C}}{k T} .
$$

1. Термоэлектрические коэффициенты для $1 \mathrm{D}$ баллистических резисторов

$$
\begin{gathered}
\mathrm{G}=\frac{2 \mathrm{q}^{2}}{\mathrm{~h}} \mathfrak{I}_{-1}\left(\eta_{\mathrm{F}}\right) ; \\
S_{T}=-\frac{k}{q} \frac{2 q^{2}}{h}\left[\mathfrak{I}_{0}\left(\eta_{F}\right)-\eta_{F} \mathfrak{I}_{-1}\left(\eta_{F}\right)\right] \\
S=-\frac{k}{q}\left[\frac{\mathfrak{I}_{0}\left(\eta_{F}\right)}{\mathfrak{I}_{-1}\left(\eta_{F}\right)}-\eta_{F}\right]
\end{gathered}
$$

$K_{0}=T\left(\frac{k}{q}\right)^{2} \frac{2 q^{2}}{h}\left[2 \mathfrak{J}_{1}\left(\eta_{F}\right)-2 \eta_{F} \mathfrak{J}_{0}\left(\eta_{F}\right)+\eta_{F}^{2} \mathfrak{J}_{-1}\left(\eta_{F}\right)\right]$;

$$
K=T\left(\frac{k}{q}\right)^{2} \frac{2 q^{2}}{h}\left[2 \mathfrak{J}_{1}\left(\eta_{F}\right)-\frac{\mathfrak{J}_{0}^{2}\left(\eta_{F}\right)}{\mathfrak{J}_{-1}\left(\eta_{F}\right)}\right] .
$$

2. Термоэлектрические коэффициенты для $1 \mathrm{D}$ диффузионных резисторов

$$
\begin{gathered}
G=\frac{2 q^{2}}{h}\left(\frac{\lambda_{0}}{L}\right) \Gamma(r+1) \mathfrak{I}_{r-1}\left(\eta_{F}\right) ; \\
S_{T}=-\frac{k}{q} \frac{2 q^{2}}{h}\left(\frac{\lambda_{0}}{L}\right) \Gamma(r+1)\left[(r+1) \mathfrak{I}_{r}\left(\eta_{F}\right)-\eta_{F} \mathfrak{I}_{r-1}\left(\eta_{F}\right)\right] ; \\
S=-\frac{k}{q}\left[\frac{(r+1) \mathfrak{I}_{r}\left(\eta_{F}\right)}{\mathfrak{I}_{r-1}\left(\eta_{F}\right)}-\eta_{F}\right] ; \\
\times\left[\Gamma(r+3) \mathfrak{J}_{r+1}\left(\eta_{F}\right)-2 \eta_{F} \Gamma(r+2) \mathfrak{I}_{r}\left(\eta_{F}\right)+\eta_{F}^{2} \Gamma(r+1) \mathfrak{I}_{r-1}\left(\eta_{F}\right)\right] ; \\
K=T\left(\frac{k}{q}\right)^{2} \frac{2 q^{2}}{h}\left(\frac{\lambda_{0}}{L}\right) \Gamma(r+2)\left[(r+2) \mathfrak{I}_{r+1}\left(\eta_{F}\right)-\frac{(r+1) \mathfrak{I}_{r}^{2}\left(\eta_{F}\right)}{\left.\mathfrak{J}_{r-1}\left(\eta_{F}\right)\right] .}\right.
\end{gathered}
$$

Проводимость $G=\sigma_{1 D} / L$ приведена в Сименсах: $\left[\sigma_{1 D}\right]=1 C м \cdot m$. Аналогично для остальных коэффициентов: $s_{T}=S_{T} L ; \quad \kappa_{0}=K_{0} L ; \quad \kappa=K L$.

3. Термоэлектрические коэффициенты для $2 \mathrm{D}$ баллистических резисторов

$$
\begin{gathered}
G=W \frac{2 q^{2}}{h} \frac{\sqrt{2 \pi m * k T}}{h} \mathfrak{I}_{-1 / 2}\left(\eta_{F}\right) ; \\
S_{T}=-W \frac{k}{q} \frac{2 q^{2}}{h} \frac{\sqrt{2 \pi m * k T}}{h}\left[\frac{3}{2} \mathfrak{I}_{1 / 2}\left(\eta_{F}\right)-\eta_{F} \mathfrak{I}_{-1 / 2}\left(\eta_{F}\right)\right] \\
S=-\frac{k}{q}\left[\frac{3 \mathfrak{I}_{1 / 2}\left(\eta_{F}\right)}{2 \mathfrak{J}_{-1 / 2}\left(\eta_{F}\right)}-\eta_{F}\right] ; \\
K_{0}=W T\left(\frac{k}{q}\right)^{2} \frac{2 q^{2}}{h} \frac{\sqrt{2 \pi m^{*} k T}}{h}\left[\frac{15}{4} \mathfrak{I}_{3 / 2}\left(\eta_{F}\right)-3 \eta_{F} \mathfrak{I}_{1 / 2}\left(\eta_{F}\right)+\eta_{F}^{2} \mathfrak{I}_{-1 / 2}\left(\eta_{F}\right)\right] ; \\
K=W T\left(\frac{k}{q}\right)^{2} \frac{2 q^{2}}{h} \frac{\sqrt{2 \pi m * k T}}{h}\left[\frac{15}{4} \mathfrak{I}_{3 / 2}\left(\eta_{F}\right)-\frac{9 \mathfrak{I}_{1 / 2}^{2}\left(\eta_{F}\right)}{4 \mathfrak{I}_{-1 / 2}\left(\eta_{F}\right)}\right]
\end{gathered}
$$

4. Термоэлектрические коэффициенты для 2D диффузионных резисторов

$$
\begin{gathered}
\mathrm{G}=\mathrm{W} \frac{2 \mathrm{q}^{2}}{\mathrm{~h}}\left(\frac{\lambda_{0}}{\mathrm{~L}}\right) \frac{\sqrt{2 \mathrm{~m} * \mathrm{kT}}}{\pi \mathrm{h}} \Gamma\left(\mathrm{r}+\frac{3}{2}\right) \mathfrak{J}_{\mathrm{r}-1 / 2}\left(\eta_{\mathrm{F}}\right) ; \\
S=-\frac{k}{q}\left[\frac{(r+3 / 2) \mathfrak{J}_{r+1 / 2}\left(\eta_{F}\right)}{\mathfrak{J}_{r-1 / 2}\left(\eta_{F}\right)}-\eta_{F}\right] ;
\end{gathered}
$$




$$
\begin{gathered}
S_{T}=-W \frac{k}{q} \frac{2 q^{2}}{h}\left(\frac{\lambda_{0}}{L}\right) \frac{\sqrt{2 m * k T}}{\pi h} \times \\
\times\left[\Gamma\left(r+\frac{5}{2}\right) \mathfrak{J}_{r+1 / 2}\left(\eta_{F}\right)-\eta_{F} \Gamma\left(r+\frac{3}{2}\right) \mathfrak{I}_{r-1 / 2}\left(\eta_{F}\right)\right] ; \\
K_{0}=W T\left(\frac{k}{q}\right)^{2} \frac{2 q^{2}}{h}\left(\frac{\lambda_{0}}{L}\right) \frac{\sqrt{2 m^{*} k T}}{\pi h} \times \\
\times\left[\Gamma\left(r+\frac{7}{2}\right) \mathfrak{J}_{r+3 / 2}\left(\eta_{F}\right)-2 \eta_{F} \Gamma\left(r+\frac{5}{2}\right) \mathfrak{J}_{r+1 / 2}\left(\eta_{F}\right)+\eta_{F}^{2} \Gamma\left(r+\frac{3}{2}\right) \mathfrak{I}_{r-1 / 2}\left(\eta_{F}\right)\right] ; \\
K=W T\left(\frac{k}{q}\right)^{2} \frac{2 q^{2}}{h}\left(\frac{\lambda_{0}}{L}\right) \frac{\sqrt{2 m * k T}}{\pi h} \Gamma\left(r+\frac{5}{2}\right) \times \\
\times\left[\left(r+\frac{5}{2}\right) \mathfrak{J}_{r+3 / 2}\left(\eta_{F}\right)-\frac{\left(r+\frac{3}{2}\right) \mathfrak{J}_{r+1 / 2}^{2}\left(\eta_{F}\right)}{\mathfrak{J}_{r-1 / 2}\left(\eta_{F}\right)}\right] .
\end{gathered}
$$

Проводимость $G=\sigma_{2 D} W / L$ приведена в $\mathrm{Cu}$ менсах: $\left[\sigma_{2 D}\right]=1 C$. Аналогично для остальных коэффициентов:

$$
s_{T}=S_{T} L / W ; \quad \kappa_{0}=K_{0} L / W ; \quad \kappa=K L / W .
$$

5. Термоэлектрические коэффициенты для $3 \mathrm{D}$ баллистических резисторов

$$
\begin{aligned}
& G=A \frac{2 q^{2}}{h} \frac{m^{*} k T}{2 \pi \hbar^{2}} \mathfrak{J}_{0}\left(\eta_{F}\right) ; \\
& S_{T}=-A \frac{k}{q} \frac{2 q^{2}}{h} \frac{m^{*} k T}{2 \pi \hbar^{2}}\left[2 \mathfrak{I}_{1}\left(\eta_{F}\right)-\eta_{F} \mathfrak{I}_{0}\left(\eta_{F}\right)\right] ; \\
& S=-\frac{k}{q}\left[\frac{2 \mathfrak{I}_{1}\left(\eta_{F}\right)}{\mathfrak{J}_{0}\left(\eta_{F}\right)}-\eta_{F}\right] \\
& K_{0}=A T\left(\frac{k}{q}\right)^{2} \frac{2 q^{2}}{h} \frac{m^{*} k T}{2 \pi \hbar^{2}}\left[6 \mathfrak{I}_{2}\left(\eta_{F}\right)-4 \eta_{F} \mathfrak{I}_{1}\left(\eta_{F}\right)+\eta_{F}^{2} \mathfrak{J}_{0}\left(\eta_{F}\right)\right] \text {; } \\
& K=A T\left(\frac{k}{q}\right)^{2} \frac{2 q^{2}}{h} \frac{m^{*} k T}{2 \pi \hbar^{2}}\left[6 \mathfrak{I}_{2}\left(\eta_{F}\right)-\frac{4 \mathfrak{I}_{1}^{2}\left(\eta_{F}\right)}{\mathfrak{I}_{0}\left(\eta_{F}\right)}\right] .
\end{aligned}
$$

6. Термоэлектрические коэффициенты для 3D диффузионных резисторов

$$
\begin{gathered}
G=A \frac{2 q^{2}}{h}\left(\frac{\lambda_{0}}{L}\right) \frac{m^{*} k T}{2 \pi \hbar^{2}} \Gamma(r+2) \mathfrak{I}_{r}\left(\eta_{F}\right) ; \\
S=-\frac{k}{q}\left[\frac{(r+2) \mathfrak{I}_{r+1}\left(\eta_{F}\right)}{\mathfrak{J}_{r}\left(\eta_{F}\right)}-\eta_{F}\right] ; \\
\mathrm{S}_{\mathrm{T}}=-\mathrm{A} \frac{\mathrm{k}}{\mathrm{q}} \frac{2 \mathrm{q}^{2}}{\mathrm{~h}}\left(\frac{\lambda_{0}}{\mathrm{~L}}\right) \frac{\mathrm{m}^{*} \mathrm{kT}}{2 \pi \hbar^{2}}\left[\Gamma(\mathrm{r}+3) \mathfrak{J}_{\mathrm{r}+1}\left(\eta_{\mathrm{F}}\right)-\eta_{\mathrm{F}} \Gamma(\mathrm{r}+2) \mathfrak{I}_{\mathrm{r}}\left(\eta_{\mathrm{F}}\right)\right] ; \\
\mathrm{K}_{0}=\mathrm{AT}\left(\frac{\mathrm{k}}{\mathrm{q}}\right)^{2} \frac{2 \mathrm{q}^{2}}{\mathrm{~h}}\left(\frac{\lambda_{0}}{\mathrm{~L}}\right) \frac{\mathrm{m}^{*} \mathrm{kT}}{2 \pi \hbar^{2}} \times \\
\times\left[\Gamma(\mathrm{r}+4) \mathfrak{J}_{\mathrm{r}+2}\left(\eta_{\mathrm{F}}\right)-2 \eta_{\mathrm{F}} \Gamma(\mathrm{r}+3) \mathfrak{I}_{\mathrm{r}+1}\left(\eta_{\mathrm{F}}\right)+\eta_{\mathrm{F}}^{2} \Gamma(\mathrm{r}+2) \mathfrak{I}_{\mathrm{r}}\left(\eta_{\mathrm{F}}\right)\right] ; \\
K=A T\left(\frac{k}{q}\right)^{2} \frac{2 q^{2}}{h}\left(\frac{\lambda_{0}}{L}\right) \frac{m^{*} k T}{2 \pi \hbar^{2}} \Gamma(r+3) \times \\
\times\left[(r+3) \mathfrak{J}_{r+2}\left(\eta_{F}\right)-\frac{(r+2) \mathfrak{J}_{r+1}^{2}\left(\eta_{F}\right)}{\mathfrak{J}_{r}\left(\eta_{F}\right)}\right] .
\end{gathered}
$$

Проводимость $G=\sigma_{3 D} A / L$ приведена в $C u$ менсах: $\left[\sigma_{3 D}\right]=1 C$ м / . Аналогично для остальных коэффициентов:

$$
s_{T}=S_{T} L / A ; \quad \kappa_{0}=K_{0} L / A ; \quad \kappa=K L / A .
$$

В основу настоящего обзора положены лекции Марка Лундстрома «Near-Equilibrium Transport: Fundamentals and Applications» [6] и Суприе Датты «Fundamentals of Nanoelectronics, Part I: Basic Concepts» [12], прочитанных в 2011 - 2012 годах в рамках инициативы Purdue University / nanoHUB-U [www.nanohub.org/u], а также наши статьи $[13,14]$.

\section{Благодарности}

Автор благодарен Н. Е. Кругляк за помощь в работе при подготовке рукописи к печати.

\section{Литература}

1. Кругляк, Ю. А. Обобщенная модель электронного транспорта Ландауэра-Датты-Лундстрома [Текст] / Ю. А. Кругляк // Nanosystems, Nanomaterials, Nanotechnologies. - 2013. - T. 11, № 3. - C. 519 - 549. Erratum: ibid. - 2014. - T. 12, № 2. - C. 415.

2. Кругляк, Ю. А. От баллистической проводимости к диффузионной в транспортной модели Ландауэра-ДаттыЛундстрома [Текст] / Ю. А. Кругляк // Nanosystems, Nanomaterials, Nanotechnologies. - 2013. - T. 11, № 4. C. 655-677.

3. Кругляк, Ю. А. Термоэлектрические явления и устройства в концепции Ландауэра-Датты-Лундстрома [Текст] / Ю. А. Кругляк // ScienceRise. - 2015. - Т. 1, № 2(6). - C. 69-77. doi: 10.15587/2313-8416.2015.35891

4. Lundstom, M. Nanoscale Transistors: Physics, Modeling, and Simulation [Text] / M. Lundstom, J. Guo. - Berlin: Springer, 2006. $-218 \mathrm{p}$.

5. Kim, R. Notes on Fermi - Dirac Integrals. Third edition [Electronic resource] / R. Kim, M. S. Lundstrom. - Purdue University. - Available at: www.nanohub.org/resources/5475

6. Lundstrom, M. Near-Equilibrium Transport: Fundamentals and Applications [Electronic resource] / M. Lundstrom, C. Jeong. - Hackensack, New Jersey: World Scientific Publishing Company, 2013. - Available at: www.nanohub.org/resources/11763

7. Sommerfeld, A. An electronic theory of the metals based on Fermi's statistics [Text] / A. Sommerfeld // Journal of Physics. - 1928. - Vol. 47, Issue .- P. 1.

8. Ашкрофт, Н. Физика твердого тела [Текст] / Н. Ашкрофт, Н. Мермин. - М: Мир, 1979. - 486 с.

9. Geballe, T. N. Seebeck Effect in Germanium [Text]/ T. N. Geballe, G. W. Hull // Physical Review. - 1954. Vol. 94, Issue 5. - $\quad$ P. 1134-1140. doi: 10.1103/physrev.94.1134

10. Pierret, R. F. Semiconductor Device Fundamentals [Text] / R. F. Pierret. - Reading, MA: Addison-Wesley, 1996. $-792 \mathrm{p}$.

11. Kim, R. S. Physics and Simulation of Nanoscale Electronic and Thermoelectric Devices [Text] / R. S. Kim. West Lafayette: Purdue University, 2011. - 218 p.

12. Supriyom D. Lessons from Nanoelectronics: A New Perspective on Transport [Text] / D. Supriyo. - Hackensack, New Jersey: World Scientific Publishing Company, 2012. 473 p. - Available at: www.nanohub.org/courses/FoN1

13. Кругляк, Ю. О. Уроки наноелектроніки. Термоелектричні явища в концепції «знизу - вгору» [Текст] / Ю. О. Кругляк, Н. Ю. Кругляк, М. В. Стріха // Sensor Electronics Microsys. Tech. - 2013. - T. 13, № 1. C. 6-21.

14. Кругляк, Ю. А. Уроки наноэлектроники. 4. Термоэлектрические явления в концепции «снизу - вверх» [Текст] / Ю. А. Кругляк // Физическое образование в вузах. -2013 . - Т. 19, № 4. - С. 70-85.

\section{References}

1. Kruglyak, Yu. A. (2013). The Generalized LandauerDatta-Lunstrom Electron Transport Model. Nanosystems, 
Nanomaterials, Nanotechnologies, 11 (3), 519-549. Erratum: ibid, (2014)., 12 (2), 415.

2. Kruglyak, Yu. A. (2013). From Ballistic Conductivity to Diffusional in the Landauer-Datta-Lunstrom. Transport Model, Nanosystems, Nanomaterials, Nanotechnologies, 11 (4), 655-677.

3. Kruglyak, Yu. A. (2015). Thermoelectric phenomena and devices in the Landauer-Datta-Lunstrom approach. ScienceRise, 1/2(6), 69-77.

doi: $10.15587 / 2313-8416.2015 .35891$

4. Lundstom, M., Guo, J. (2006). Nanoscale Transistors: Physics, Modeling, and Simulation. Berlin: Springer, 218.

5. Kim, R., Lundstrom, M. S. Notes on Fermi - Dirac Integrals. Purdue University. Available at: www.nanohub.org/resources/5475

6. Lundstrom, M., Jeong, C. (2013). Near-Equilibrium Transport: Fundamentals and Applications. Hackensack, New Jersey: World Scientific Publishing Company. Available at: www.nanohub.org/resources/11763

7. Sommerfeld, A. (1928). An electronic theory of the metals based on Fermi's statistics. Journal of Physics, 47 (1), 1.
8. Ashcroft, N. W., Mermin, N. D. (1979). Solid State Physics (Philadelphia: Suanders College, 486.

9. Geballe, T. N., Hull, G. W. (1954). Seebeck Effect in Germanium, Physical Review, 94 (5), 1134-1140. doi: 10.1103/physrev.94.1134

10. Pierret, R. F. (1996). Semiconductor Device Fundamentals. Reading, MA: Addison-Wesley, 792.

11. Kim, R. S. (2011). Physics and Simulation of Nanoscale Electronic and Thermoelectric Devices. West Lafayette: Purdue University, 218.

12. Supriyo, D. (2012). Lessons from Nanoelectronics: A New Perspective on Transport. Hackensack, New Jersey: World Scientific Publishing Company, 473. Available at: www.nanohub.org/courses/FoN1

13. Kruglyak, Yu. A., Kruglyak, N. Yu., Strikha, M. V. (2013). Lessons of nanoelectronics. Thermoelectric phenomena in «bottom - up» approach, Sensor Electronics Microsys. Tech., 13 (1), 6-21.

14. Kruglyak, Yu. A. (2013). Lessons of nanoelectronics. 4. Thermoelectric phenomena in «bottom - up» approach. Physics in Higher Education, 19 (4), 70-85.

Рекомендовано до публікації д-р фіз.-мат. наук Глуиков О. В. Дата надходження рукопису 15.12.2014

Кругляк Юрій Олексійович, доктор хімічних наук, профессор, кафедра інформаційних технологій, Одеський державний екологічний університет, вул. Львівська, 15, м. Одеса, Україна, 65016

E-mail: quantumnet@yandex.ua

УДК 537.622: 544.227: 621.318.1

DOI: 10.15587/2313-8416.2015.36410

\section{МАГНИТНАЯ И ЭЛЕКТРОННО-ИОННАЯ СТРУКТУРА MnZn-ФЕРРИТОВ В ПРИПОВЕРХНОСТНЫХ СЛОЯХ, ОБЕДНЕННЫХ И ОБОГАЩЕННЫХ ЦИНКОМ}

\section{(C) А. В. Копаев, В. В. Мокляк, В. С. Бушкова}

Исследованы процессы, происходящчие на атомном уровне вблизи поверхности феррита $\mathrm{Mn}_{0.6} \mathrm{Zn}_{0.3} \mathrm{Co}_{0.05} \mathrm{Fe}_{2.05} \mathrm{O}_{4}$, полученных в различных условиях - стандартной и специиальной атмосфере. Предлагаемый способ насыщения циинком поверхностных слоев феррита частично восстанавливает не только химический состав, но и магнитную структуру вблизи поверхности ферритовых изделий

Ключевые слова: марганеи-иинковый феррит, насыщение ичинко, рентгеновская спектроскопия поглощения, мессбауэровская спектроскопия

It is investigated the processes occurring at the atomic level near the surface of the ferrite $\mathrm{Mn}_{0.6} \mathrm{Zn}_{0.3} \mathrm{Co}_{0.05} \mathrm{Fe}_{2.05} \mathrm{O}_{4}$, obtained in different conditions - standard and special atmospheres. The proposed method of Zn-saturation of the surface layers of ferrite partially recovers not only the chemical composition but also the magnetic structure near the surface of the ferrite products

Keywords: manganese-zinc ferrite, saturation with zinc, X-ray absorption spectroscopy, Mössbauer spectroscopy

\section{1. Введение}

Среди материалов с высокой магнитной проницаемостью марганец-цинковые ферриты со структурой шпинели занимают важное место. Они имеют высокое электрическое сопротивление и низкие потери энергии в переменных магнитных полях. Их технология сравнительно проста и хорошо освоена, однако пока еще не оптимальна.

\section{публикаций \\ 2. Постановка проблемы и анализ \\ Одна из проблем $[1,2]$, которая пока еще не} полностью решена, состоит в следующем. Известно, что цинк, входящий в состав феррита, при температурах синтеза выше $1000{ }^{\circ} \mathrm{C}$ обладает высокой упруго- стью паров над поверхностью твердой фазы $[3,4]$. При этом вблизи поверхности образцов материал обедняется цинком. Отклонения элементного состава в приповерхностном слое ферритов приводит к неконтролируемым изменениям и к ухудшению электромагнитных параметров изделий.

Мы сопоставили табличные данные из $[3,4]$ и представили их в виде графиков зависимости логарифма скорости испарения цинка от обратной температуры синтеза при данном давлении кислорода (рис. 1). Как видно из рисунка, эта зависимость практически линейная для данного химического состава феррита. Анализ показывает, что скорость испарения цинка тем выше, чем больше температура и концентрация цинка в твердофазной матрице, а также чем меньше парциальное 